\title{
LAGRANGE MULTIPLIERS FOR HIGHER ORDER ELLIPTIC OPERATORS
}

\author{
Carlos Zuppa ${ }^{1}$
}

\begin{abstract}
In this paper, the Babuška's theory of Lagrange multipliers is extended to higher order elliptic Dirichlet problems. The resulting variational formulation provides an efficient numerical squeme in meshless methods for the approximation of elliptic problems with essential boundary conditions.
\end{abstract}

Mathematics Subject Classification. 41A10, 41A17, 65N15, 65N30.

Received: April 5, 2004.

\section{INTRODUCTION}

In spite of the great success of the finite element method as effective numerical tool for the solution of boundary-value problems in complex domains, there has been a growing interest in meshless methods over the last decade. The automatic generation of 3-D meshes presents significant difficulties in the analysis of engineering systems and the development of techniques which do not require the generation of a mesh is very appealing. In meshless method $h-p$ (spectral) types of approximations are built around a collection of nodes sprinkled within the domain on which a boundary-value problem has been posed. Associated with each node, there is an open set that provides the support for the approximation basis functions built around the node. The boundary-value problem is then solved using these $h-p$ functions and a Galerkin method. A number of methods have been proposed so far including the smooth particle hydrodynamics (SHP) method, the diffuse element method (DEM), the element free Galerkin (EFG) method, the reproducing kernel particle method (RKPM), the moving least-squares reproducing kernel method (MLSRK), the $h$ - $p$ clouds method (HPC), the partition of unity finite element method (PUFEM), among others. Extensive reviews of meshless methods can be found in $[4,8,10]$.

One major difficulty with almost all meshless methods is the imposition of essential boundary conditions. Nonetheless, there are many ways to overcome this problem. Most meshless methods use Lagrange multipliers or penalty methods to impose essential boundary conditions, at least for second order elliptic problems. The objective of this paper is to present the Lagrange multipliers method for higher order elliptic problems.

A brief outline of the paper is as follows. In Section 2 we shall establish our main settings. In Section 3, we remind the basic facts on coercive bilinear forms that will be used in this work. Section 4 is devoted to our main results on Babuška's theory of Lagrange multipliers. In the last section we shall make a few comments on the numerical implementation of this methodology in the context of the $h-p$ clouds meshless method. Detailed implementations and extensive numerical experiments will be given in a forthcoming paper [15].

\footnotetext{
Keywords and phrases. Elliptic operators, Dirichlet boundary-value problem, Lagrange multipliers.

1 Departamento de Matemáticas, Universidad Nacional de San Luis, Chacabuco y Pedernera, 5700, San Luis, Argentina. zuppa@unsl.edu.ar
} 


\section{Formulation of the problem}

We suppose $\Omega$ is a smooth, open, bounded subset of $\mathbb{R}^{n}$ with a smooth boundary $\partial \Omega$, and we consider the boundary-value problem

$$
\begin{array}{lll}
A u=f & \text { in } \Omega & \\
\Psi_{k}=g_{k} & \text { on } \partial \Omega & 0 \leq k \leq m-1 .
\end{array}
$$

Here, $A$ is a $2 m$ th-order regularly elliptic real differential operator of the form

$$
A u=\sum_{|\alpha|,|\beta| \leq m}(-1)^{|\alpha|} D^{\alpha}\left(a_{\alpha \beta} D^{\beta} u\right),
$$

and the collection of boundary operators $\Psi=\left\{\Psi_{k}\right\}_{k=0}^{m-1}$ form a Dirichlet system of order $m$ on $\partial \Omega$ (see $\left.[5,9,12]\right)$. To simplify, we assume smooth coefficients: $a_{\alpha \beta}, a_{0} \in C^{\infty}(\bar{\Omega}), b_{k \alpha} \in C^{\infty}(\partial \Omega)$, where $b_{k \alpha}$ are the coefficients of the boundary operator $\Psi_{k}$.

Remark 2.1. The assumption on the boundary $\partial \Omega$ is certainly a simplification by which almost all theories begin. In practical applications this is not generally the case and it is well known that singular points in $\partial \Omega$ pose additional problems.

We associate to the operator $A$ the bilinear form

$$
\mathcal{B}(u, v):=\sum_{|\alpha|,|\beta| \leq m} \int_{\Omega} a_{\alpha \beta}(x) D^{\beta} u(x) D^{\alpha} v(x) \mathrm{d} x, \quad \forall u, v \in C^{\infty}(\bar{\Omega}) .
$$

There exists (e.g., see [9]) a complementary system of boundary operators $\Phi=\left\{\Phi_{k}\right\}_{k=0}^{m-1}$, where the order of $\Phi_{k}$ is $2 m-1-k, k=$ order of $\Psi_{k}$, such that the Green's formula holds for every $u, v \in C^{\infty}(\bar{\Omega})$ :

$$
\int_{\Omega} A u v \mathrm{~d} x=\mathcal{B}(u, v)-\sum_{k=0}^{m-1} \int_{\partial \Omega} \Phi_{k} u \Psi_{k} v \mathrm{~d} s
$$

The bilinear form $\mathcal{B}$ can be extended to a continuous bilinear form over $H^{m}(\Omega) \times H^{m}(\Omega)$. In particular, there exists a constant $C_{\mathcal{B}}$ such that

$$
|\mathcal{B}(u, v)| \leq C_{\mathcal{B}}\|u\|_{H^{m}(\Omega)}\|v\|_{H^{m}(\Omega)}, \quad \forall u, v \in H^{m}(\Omega) .
$$

Similarly, the boundary operators $\Psi_{k}$ can be extended to continuous linear operators

$$
\Psi_{k}: H^{m}(\Omega) \rightarrow H^{m-k-1 / 2}(\partial \Omega)
$$

Given

we define

$$
\mathbf{g}=\left(g_{k}\right) \in Q=\prod_{k=0}^{m-1} H^{m-k-1 / 2}(\partial \Omega)
$$

$$
H_{g}^{m}(\Omega):=\left\{u: u \in H^{m}(\Omega) ; B_{k}(u)=g_{k} ; 0 \leq k \leq m-1\right\} .
$$

If $f \in L^{2}(\Omega)$, the variational solution of problem (2.1) is to find $\widehat{u} \in H_{g}^{m}(\Omega)$, such that

$$
\mathcal{B}(\widehat{u}, v)-\sum_{k=0}^{m-1} \int_{\partial \Omega} \Phi_{k} \widehat{u} g_{k} \mathrm{~d} s=\int_{\Omega} f v \mathrm{~d} x, \quad \forall v \in H_{g}^{m}(\Omega) .
$$


One problem is that boundary operators $\Phi_{k}$ cannot be extended to continuous operators:

$$
\Phi_{k}: H^{m}(\Omega) \rightarrow H^{m-k-1 / 2}(\partial \Omega)^{\prime} .
$$

A smaller space would be needed and it so happens that this smaller space is precisely the space $H^{m}(\Omega, \Phi)$ which is the completion of $C^{\infty}(\bar{\Omega})$ in the norm

$$
\|u\|_{m, \Phi}^{2}:=\|u\|_{H^{m}(\Omega)}^{2}+\sum_{k=0}^{m-1}\left\|\Phi_{k} u\right\|_{H^{m-k-1 / 2}(\partial \Omega)^{\prime}} .
$$

Another drawback in practical approximation of the solution of (2.4) is the fulfillment of the boundary conditions. In most meshless methods this is a major drawback. One way to avoid these difficulties is to use the method of variational principles in conjunction with the technique of Lagrange multipliers. This methodology was introduced by Babuška $[2,3]$ in case $m=1$ and was extensively used in meshless methods for solving PDEs (e.g., see $[7,8])$.

The aim of this paper is to developed Babuška's theory of Lagrange multipliers for $m>1$, at least when the form $\mathcal{B}$ is $\left(H^{m}(\Omega), H^{0}(\Omega)\right)$-coercive. As a by-product, we obtain that the solution $\widehat{u}$ of the problem (2.4) effectively exists in $H^{m}(\Omega, \Phi)$. Thus, the theory of Lagrange multipliers provides not only an efficient numerical method but also a regularity result.

A weak solution $\widetilde{u}$ of problem $(2.1)$ can be obtained in the space $\widetilde{H}^{m, 2 m}(\Omega) \subset H^{m}(\Omega)$, defined as the completion of $C^{\infty}(\bar{\Omega})$ in the norm:

$$
\|u\|_{\widetilde{H}^{m, 2 m}(\Omega)}^{2}:=\|u\|_{H^{m}(\Omega)}^{2}+\sum_{k=0}^{2 m-1}\left\|D_{n}^{k} u\right\|_{H^{m-k-1 / 2}(\partial \Omega)},
$$

where $D_{n}^{k}:=\partial^{k} / \partial n^{k}$ is the $k$ th-normal derivative (see Berezanskii [5] and also [11,14]). Of course, we must have $\widehat{u}=\widetilde{u}$ and $\widehat{u} \in H^{m}(\Omega, \Phi) \cap \widetilde{H}^{m, 2 m}(\Omega)$. However, we cannot prove directly that $\Phi_{k} \widetilde{u} \in H^{m-k-1 / 2}(\partial \Omega)^{\prime}$, $k=0, \ldots, m-1$. Then, our result is in fact different, except in case $m=1$.

\section{Coercive BILINEAR Forms}

It is well known that coerciveness is a crucial ingredient in the variational theory of differential operators.

Definition 3.1. The bilinear form $(2.2)$ is $\left(H^{m}(\Omega), H^{0}(\Omega)\right)$-coercive if there exists a constant $\gamma_{0}$ such that for every $\gamma>\gamma_{0}$ the form $\mathcal{B}_{\gamma}$ defined by

$$
\mathcal{B}_{\gamma}(u, v):=\mathcal{B}(u, v)+\gamma \int_{\Omega} u v \mathrm{~d} x, \quad u, v \in H^{m}(\Omega)
$$

is $H^{m}(\Omega)$-elliptic. That is, there exists a constant $c_{\gamma}>0$ such that

$$
\mathcal{B}_{\gamma}(u, u) \mid \geq c_{\gamma}\|u\|_{H^{m}(\Omega)}, \quad \forall u \in H^{m}(\Omega) .
$$

If $m=1$, Garding's inequality implies that $(2.2)$ is $\left(H^{m}(\Omega), H^{0}(\Omega)\right)$-coercive [6]. This is not always the case when $m>1$. In the model for the plate bending for example, the bilinear form defined on $H^{2}(\Omega)$ is given by

$$
\mathcal{B}(u, v)=\int_{\Omega} \Delta u \Delta v-(1-\nu)\left(2 u_{x x} v_{y y}+2 u_{y y} v_{x x}-4 u_{x y} v_{x y}\right) \mathrm{d} x \mathrm{~d} y
$$

where $\nu$ is a physical constant known as Poisson's ratio. $\mathcal{B}$ is known to be $\left(H^{m}(\Omega), H^{0}(\Omega)\right)$-coercive for all $-3<\nu<1[1]$. For $\nu=1, \mathcal{B}$ cannot be $\left(H^{m}(\Omega), H^{0}(\Omega)\right)$-coercive since $\mathcal{B}(u, v)$ vanishes for all harmonic $u, v$. 
Conditions for $\left(H^{m}(\Omega), H^{0}(\Omega)\right)$-coerciveness of bilinear forms associated with higher order elliptic operators are given in $[12,13]$.

We summarize our major use of $H^{m}(\Omega)$-elliptic bilinear forms in the following result.

Proposition 3.2. Let $\mathcal{B}_{\gamma}$ be $H^{m}(\Omega)$-elliptic. Then, the Neumann problem is solvable. That is, for every

$$
\lambda=\left(\lambda_{k}\right) \in Q^{\prime}=\prod_{k=0}^{m-1} H^{m-k-1 / 2}(\partial \Omega)^{\prime},
$$

there exists a unique $u_{\lambda} \in H^{m}(\Omega)$, such that

$$
\mathbf{B}_{\gamma}\left(v, u_{\lambda}\right)=\sum_{k=0}^{m-1}\left\langle\lambda_{k}, \Psi_{k} v\right\rangle, \quad \forall v \in H^{m}(\Omega) .
$$

Furthermore, there exists a constant $c=c(\mathcal{B}, \Psi, \gamma)$ such that

$$
\sum_{k=0}^{m-1}\left\|\lambda_{k}\right\|_{H^{m-k-1 / 2}(\partial \Omega)^{\prime}}^{2} \leq c\left(\sum_{k=0}^{m-1}\left\langle\lambda_{k}, \Psi_{k} u_{\lambda}\right\rangle\right), \quad \forall \lambda \in Q^{\prime} .
$$

Proof. The first assertion is an easy consequence of Hilbert space theory. For the second one, we note first that it suffices to consider the case $\lambda=\left(0, \ldots, 0, \lambda_{k}, 0, \ldots, 0\right)$, and we assume this condition.

There exists a continuous linear operator

$$
\phi_{k}: H^{m-k-1 / 2}(\partial \Omega) \rightarrow H^{m}(\Omega)
$$

such that $\Psi_{k}\left(\phi_{k}(w)\right)=w$ and a constant $c_{k}^{\prime}$ which satisfies

$$
\left\|\phi_{k}(w)\right\|_{H^{m}(\Omega)} \leq c_{k}^{\prime}\|w\|_{H^{m-k-1 / 2}(\partial \Omega)}
$$

for every $w \in H^{m-k-1 / 2}(\partial \Omega)$ (see [12], p. 157). Thus, if we replace $v$ by $\phi_{k}(w), w \in H^{m-k-1 / 2}(\partial \Omega)$ in $(3.2)$, we obtain

Clearly,

$$
\mathcal{B}_{\gamma}\left(\phi_{k}(w), u_{\lambda}\right)=\left\langle\lambda_{k}, w\right\rangle .
$$

$$
\left|\mathcal{B}_{\gamma}\left(\phi_{k}(w), u_{\lambda}\right)\right| \leq \beta\left\|u_{\lambda}\right\|_{H^{m}(\Omega)}\left\|\phi_{k}(w)\right\|_{H^{m}(\Omega)}
$$

or

$$
\left|\left\langle\lambda_{k}, w\right\rangle\right| \leq c_{k}^{\prime} \beta\left\|u_{\lambda}\right\|_{H^{m}(\Omega)}\|w\|_{H^{m-k-1 / 2}(\partial \Omega)} .
$$

In what follows we shall consider only $w \neq 0$. Hence,

$$
\begin{aligned}
\left\|\lambda_{k}\right\|_{H^{m-k-1 / 2}(\partial \Omega)^{\prime}} & =\sup _{w \in H^{m-k-1 / 2}(\partial \Omega)} \frac{\left|\left\langle\lambda_{k}, w\right\rangle\right|}{\|w\|_{H^{m-k-1 / 2}(\partial \Omega)}} \\
& \leq c_{k}^{\prime} \beta\left\|u_{\lambda}\right\|_{H^{m}(\Omega)} .
\end{aligned}
$$

On the other hand,

Then, we have

$$
\left\|u_{\lambda}\right\|_{H^{m}(\Omega)}^{2} \leq \alpha^{-1} \mathcal{B}_{\gamma}\left(u_{\lambda}, u_{\lambda}\right)=\alpha^{-1}\left\langle\lambda_{k}, \Psi_{k} u_{\lambda}\right\rangle .
$$

$$
\left\|\lambda_{k}\right\|_{H^{m-k-1 / 2}(\partial \Omega)^{\prime}}^{2} \leq\left(\left(c_{k}^{\prime} \beta\right)^{2} \alpha^{-1}\right)\left\langle\lambda_{k}, \Psi_{k} u_{\lambda}\right\rangle .
$$

The proof of the proposition is finished by setting $c=\left(\max _{0 \leq k<m}\left(c_{k}^{\prime}\right)^{2}\right) \beta^{2} \alpha^{-1}$. 
A sufficient condition on bilinear forms to guarantee the existence of the solution for the Lagrange multiplier method is given by the generalized Lax-Milgram theorem on weakly coercive forms $[2,3,9,12]$.

Theorem 3.3. Let $\mathcal{U}$ and $\mathcal{V}$ be two real Hilbert spaces, and let $\widetilde{\mathcal{B}}: \mathcal{U} \times \mathcal{V} \rightarrow \mathbb{R}$ be a continuous, weakly coercive, bilinear form; i.e., let $\widetilde{B}$ be such that the following conditions hold:

$$
\begin{array}{ll}
\text { (i) } & |\widetilde{\mathcal{B}}(u, v)| \leq M\|u\|_{\mathcal{U}}\|v\|_{\mathcal{V}} \quad \forall u \in \mathcal{U} \quad \text { and } \forall v \in \mathcal{V}, \\
\text { (ii) } & \inf _{u \in \mathcal{U},\|u\|_{\mathcal{U}}=1} \sup _{v \in \mathcal{V},\|v\|_{\mathcal{V}} \leq 1}|\widetilde{\mathcal{B}}(u, v)| \geq \gamma>0 \\
\text { (iii) } & \sup _{u \in \mathcal{U}}|\widetilde{\mathcal{B}}(u, v)|>0 \quad v \neq 0
\end{array}
$$

where $M$ and $\gamma$ are finite positive constants. Then, if $F$ is a continuous linear functional on $\mathcal{V}$ (i.e., $F \in \mathcal{V}^{\prime}$ ), there exists a unique element $u^{*} \in \mathcal{U}$ such that

$$
\widetilde{\mathcal{B}}\left(u^{*}, v\right)=F(v) \quad \forall v \in \mathcal{V}
$$

and

$$
\left\|u^{*}\right\|_{\mathcal{U}} \leq \frac{1}{\gamma}\|F\|_{\mathcal{V}^{\prime}}
$$

\section{LAGRANGE MULTIPLIERS FOR HIGHER ORDER OPERATORS}

Let

$$
\mathcal{U}=H^{m}(\Omega) \times \prod_{k=0}^{m-1} H^{m-k-1 / 2}(\partial \Omega)^{\prime} .
$$

We introduce the following norm on $\mathcal{U}$ :

$$
\|(u, \lambda)\|_{\mathcal{U}}^{2}=\|u\|_{H^{m}(\Omega)}^{2}+\sum_{k=0}^{m-1}\left\|\lambda_{k}\right\|_{H^{m-k-1 / 2}(\partial \Omega)^{\prime}}^{2} .
$$

In order to simplify writing, we denote the natural duality pairing in $Q^{\prime} \times Q$ with $\langle\langle\cdot, \cdot\rangle\rangle$. Also, for $w \in H^{m}(\Omega)$, $\Psi w \in Q$ is the $m$-uple $\left(\Psi_{k} w\right)_{k=0}^{m-1}$ and so on.

We now define the continuous symmetric bilinear form $\widetilde{\mathcal{B}}: \mathcal{U} \times \mathcal{U} \rightarrow \mathbb{R}^{n}$ by the formula:

$$
\widetilde{\mathcal{B}}((u, \lambda),(v, \mu)):=\mathcal{B}(u, v)-\langle\langle\mu, \Psi u\rangle\rangle-\langle\langle\lambda, \Psi v\rangle\rangle .
$$

Our main result is:

Theorem 4.1. If $\mathcal{B}$ is $\left(H^{m}(\Omega), H^{0}(\Omega)\right)$-coercive then the form $\widetilde{\mathcal{B}}$ is weakly coercive.

Proof. All we have to do is to almost mimic word to word Babuška's proof in $[2,3]$. Let $\gamma$ be such that the bilinear form

$$
\mathcal{B}_{\gamma}(u, v)=\mathcal{B}(u, v)+\gamma u v
$$

is $H^{m}(\Omega)$-elliptic.

Given $(u, \lambda) \in \mathcal{U}$ and $\left(u-z_{1}+z_{2}, \mu\right) \in \mathcal{U}$, we have

$$
\begin{gathered}
\widetilde{\mathcal{B}}\left((u, \lambda),\left(u-z_{1}+z_{2}, \mu\right)\right)=\mathcal{B}_{\gamma}(u, u)-\mathcal{B}_{\gamma}\left(u, z_{1}\right)+\int_{\Omega}\left(a_{0}-\gamma\right) u\left(u-z_{1}\right) \mathrm{d} x+\mathcal{B}\left(u, z_{2}\right) \\
-\left\langle\left\langle\lambda, \Psi\left(u-z_{1}+z_{2}\right)\right\rangle\right\rangle-\left\langle\left\langle\mu, \Psi_{k} u\right\rangle\right\rangle .
\end{gathered}
$$


By selecting $z_{1} \in H^{m}(\Omega)$ as the solution of the Neumann problem

$$
\mathcal{B}_{\gamma}\left(v, z_{1}\right)=\langle\langle\lambda, \Psi v\rangle\rangle, \quad \forall v \in H^{m}(\Omega),
$$

we have

$$
\left\|z_{1}\right\|_{H^{m}(\Omega)} \leq C\|\lambda\|_{Q}
$$

Now, we consider the adjoint operator $A^{*}$. There exists a Dirichlet system $\widetilde{\Psi}=\left\{\widetilde{\Psi}_{k}\right\}_{k=0}^{m-1}$ and a complementary system of boundary operators $\Gamma=\left\{\Gamma_{k}\right\}_{k=0}^{m-1}$, where the order of $\Gamma_{k}$ is $2 m-1-k$, such that the Green's formula holds:

$$
\int_{\Omega} u A^{*} v \mathrm{~d} x=\mathcal{B}(u, v)-\sum_{k=0}^{m-1} \int_{\partial \Omega} \widetilde{\Psi}_{k} u \Gamma_{k} v \mathrm{~d} s .
$$

Let $z_{2}$ be the solution of the elliptic problem with homogeneous boundary conditions:

$$
\begin{aligned}
& A^{*} w=-\left(a_{0}-\gamma\right)\left(u-z_{1}\right), \\
& \widetilde{\Psi}_{k} w=0, \quad 0 \leq k \leq m-1 .
\end{aligned}
$$

Also, it may be proved that

$$
\Psi_{k} z_{2}=0, \quad 0 \leq k \leq m-1 .
$$

By regularity theory (see [12]), there exists a constant $c_{(A, \Phi)}$ such that

$$
\left\|z_{2}\right\|_{H^{3 m}(\Omega)} \leq c_{(A, \Psi)}\left\|u-z_{1}\right\|_{H^{m}(\Omega)} .
$$

Hence, there exists a constant $\widetilde{c}_{(A, \Phi, \gamma)}$ such that

$$
\left\|z_{2}\right\|_{H^{3 m}(\Omega)} \leq \widetilde{c}_{(A, \Psi, \gamma)}\|(u, \lambda)\|_{\mathcal{U}}
$$

On the other hand,

$$
\mathcal{B}\left(u, z_{2}\right)=-\int_{\Omega}\left(a_{0}-\gamma\right) u\left(u-z_{1}\right) \mathrm{d} x+\left\langle\left\langle\Gamma z_{2}, \widetilde{\Psi} u\right\rangle\right\rangle .
$$

Taking into account that $\Psi_{k} z_{2}=0,0 \leq k \leq m-1$, (4.1) can be rewritten

$$
\widetilde{\mathcal{B}}\left((u, \lambda),\left(u-z_{1}+z_{2}, \mu\right)\right)=\mathcal{B}_{\gamma}(u, u)+\left\langle\left\langle\lambda, \Psi z_{1}\right\rangle\right\rangle-\langle\langle\mu, \Psi u\rangle\rangle-\langle\langle 2 \lambda, \Psi u\rangle\rangle-\left\langle\left\langle\Gamma z_{2}, \widetilde{\Psi} u\right\rangle\right\rangle .
$$

Let $\phi: \prod_{k=0}^{m-1} H^{m-k-1 / 2}(\partial \Omega) \rightarrow H^{m}(\Omega)$ be a linear continuous functional such that

$$
\Psi(\phi(w))=w, \quad \forall w \in \prod_{k=0}^{m-1} H^{m-k-1 / 2}(\partial \Omega) .
$$

We define $\mu \in\left(\prod_{k=0}^{m-1} H^{m-k-1 / 2}(\partial \Omega)\right)^{\prime}$ by

$$
\langle\langle\mu, w\rangle\rangle:=\langle\langle 2 \lambda, w\rangle\rangle+\left\langle\left\langle\Gamma z_{2}, \widetilde{\Psi} \phi w\right\rangle\right\rangle .
$$

Hence,

$$
\widetilde{\mathcal{B}}\left((u, \lambda),\left(u-z_{1}+z_{2}, \mu\right)\right)=\mathcal{B}_{\gamma}(u, u)+\left\langle\left\langle\lambda, \Psi z_{1}\right\rangle\right\rangle ;
$$

and, therefore, by (3.3) and $H^{m}$-ellipticity, we have

$$
\begin{aligned}
\widetilde{\mathcal{B}}\left((u, \lambda),\left(u-z_{1}+z_{2}, \mu\right)\right) & \geq \alpha\|u\|_{H^{m}(\Omega)}^{2}+c^{-1}\left(\sum_{k=0}^{m-1}\left\|\lambda_{k}\right\|_{H^{m-k-1 / 2}(\partial \Omega)^{\prime}}^{2}\right) \\
& \geq \min \left(\alpha, c^{-1}\right)\|(u, \lambda)\|_{\mathcal{U}}^{2}
\end{aligned}
$$


Now, applying (4.2) and (4.5) and considering the fact that $z_{2} \in H^{3 m}(\Omega)$ in order to prove that the boundary operators $\Gamma_{k}$ are continuous, it is easy to see that there exists a constant $\bar{C}$, such that

$$
\left\|u-z_{1}+z_{2}\right\|_{H^{m}(\Omega)}^{2} \leq \bar{C}^{1 / 2}\|(u, \lambda)\|_{\mathcal{U}}^{2}
$$

and

$$
\|\mu\|_{Q^{\prime}}^{2} \leq \bar{C}^{1 / 2}\|(u, \lambda)\|_{\mathcal{U}}^{2}
$$

Hence

$$
\left\|\left(u-z_{1}+z_{2}, \mu\right)\right\|_{\mathcal{U}} \leq \bar{C}\|(u, \lambda)\|_{\mathcal{U}} .
$$

Thus,

$$
\widetilde{\mathcal{B}}\left((u, \lambda),\left(u-z_{1}+z_{2}, \mu\right)\right) \geq c^{\prime \prime}\|(u, \lambda)\|_{\mathcal{U}}\left\|\left(u-z_{1}+z_{2}, \mu\right)\right\|_{\mathcal{u}}
$$

The other condition of weak coerciveness may be proved analogously.

Consequently, there exists a constant $\sigma>0$ which satisfies: for every $F \in \mathcal{U}^{\prime}$, there exists a unique element $u^{*} \in \mathcal{U}$ such that

and

$$
\widetilde{\mathcal{B}}\left(u^{*}, v\right)=F(v) \quad \forall v \in \mathcal{V}
$$

$$
\left\|u^{*}\right\|_{\mathcal{U}} \leq \frac{1}{\sigma}\|F\|_{\mathcal{V}^{\prime}}
$$

Given $f \in L^{2}(\Omega)$ and $g=\left(g_{k}\right) \in \prod_{k=0}^{m-1} H^{m-k-1 / 2}(\partial \Omega)$, the Dirichlet variational problem associated to $\widetilde{\mathcal{B}}$ is to find $(u, \lambda) \in \mathcal{U}$, such that

$$
\widetilde{\mathcal{B}}((u, \lambda),(v, \mu))=\int_{\Omega} f v \mathrm{~d} x-\langle\langle\mu, g\rangle\rangle, \quad \forall(v, \mu) \in \mathcal{U} .
$$

This is the variational formulation that can be used in a Galerkin method for numerical approximation of the solution.

The next result is a direct consequence of the above theorem.

Corollary 4.2. For every $(f, g) \in L^{2}(\Omega) \times \prod_{k=0}^{m-1} H^{m-k-1 / 2}(\partial \Omega)$, the problem (4.6) has a unique solution $\left(u_{(f, g)}, \lambda_{(f, g)}\right) \in \mathcal{U}$. Furthermore, the assignation $(f, g) \rightarrow\left(u_{(f, g)}, \lambda_{(f, g)}\right)$ defines a continuous linear operator

$$
\mathcal{V}: L^{2}(\Omega) \times \prod_{k=0}^{m-1} H^{m-k-1 / 2}(\partial \Omega) \rightarrow H^{m}(\Omega) \times \prod_{k=0}^{m-1} H^{m-k-1 / 2}(\partial \Omega)
$$

In particular, there exists a constant $C$ such that

$$
\|\mathcal{V}(f, g)\|_{\mathcal{U}}^{2} \leq C\left(\|f\|_{L^{2}(\Omega)}^{2}+\sum_{k=0}^{m-1}\left\|g_{k}\right\|_{H^{m-k-1 / 2}(\Omega)}^{2}\right) .
$$

If $(f, g) \in C^{\infty}(\bar{\Omega}) \times \prod_{k=0}^{m-1} C^{\infty}(\partial \Omega)$, it is well known that $u_{(f, g)} \in C^{\infty}(\bar{\Omega})$. Then, for every $(v, \mu) \in C^{\infty}(\bar{\Omega}) \times Q^{\prime}$, we have

$$
\begin{aligned}
\int_{\Omega} A u_{(f, g)} v \mathrm{~d} x+ & \left\langle\left\langle\Phi u_{(f, g)}-\lambda_{(f, g)}, \Psi v\right\rangle\right\rangle-\left\langle\left\langle\mu, \Psi u_{(f, g)}\right\rangle\right\rangle \\
& =\int_{\Omega} f v \mathrm{~d} x-\langle\langle\mu, g\rangle\rangle .
\end{aligned}
$$


It follows easily that $\Phi u_{(f, g)}=\lambda_{(f, g)}$ and $\Psi u_{(f, g)}=g$. Hence, since $H^{m}(\Omega, \Phi)$ is a closed subspace of $\prod_{k=0}^{m-1} H^{m-k-1 / 2}(\partial \Omega)^{\prime}$, by a density argument it may be proved that

$$
u_{(f, g)} \in H^{m}(\Omega, \Phi), \quad \forall(f, g) \in L^{2}(\Omega) \times \prod_{k=0}^{m-1} H^{m-k-1 / 2}(\partial \Omega)
$$

Corollary 4.3. Given $f \in L^{2}(\Omega)$ and $g=\left(g_{k}\right) \in \prod_{k=0}^{m-1} H^{m-k-1 / 2}(\partial \Omega)$, the Dirichlet variational problem: find $u \in H^{m}(\Omega, \Phi)$ such that, for every $v \in H^{m}(\Omega, \Phi)$,

$$
\mathcal{B}(u, v)-\langle\langle\Phi v, \Psi u\rangle\rangle-\langle\langle\Phi u, \Psi v\rangle\rangle=\int_{\Omega} f v \mathrm{~d} x-\langle\langle\Phi v, g\rangle\rangle
$$

has a unique solution $u_{(f, g)}$. Moreover, this linear assignment is continuous, i.e., there exists a constant $C>0$ such that

$$
\left\|u_{(f, g)}\right\|_{H^{m}(\Omega, \Phi)} \leq C\left(\|f\|_{L^{2}(\Omega)}+\sum_{k=0}^{m-1}\left\|g_{k}\right\|_{H^{m-k-1 / 2}(\Omega)}\right) .
$$

Remark 4.4. Taking into account that in most meshless methods the approximation functions are sufficiently smooth, (4.7) suggests a Galerkin method which does not increase the number of unknowns. It is true that this method works almost as well as the Lagrange multipliers method when the mesh size of the grid $h$ tends to 0 . However, at relatively great $h$, it produces more instable results, especially near boundary corners.

\section{NumericAl implementation}

Even though the main aim of this paper is to prove the theoretical validity of the Lagrange multipliers method for higher order elliptic systems, we shall point out several important questions related to the numerical implementation of this methodology in the context of meshless methods.

A meshless method which has very attractive features is the $h-p$ clouds of Duarte and Oden [7]. The basic idea of the method is to multiply a partition of unity by polynomials or other class of functions. The resulting functions, called $h-p$ clouds, have good properties, such as high regularity and compactness; and linear combinations of these functions can represent polynomials of any degree. This property allows the implementation of $p$ and $h-p$ adaptivity. We shall discuss the Lagrange multipliers Galerkin method for (4.6) using the families $\mathcal{F}^{0, k}(k=1,2)$ of $h$ - $p$ clouds functions of Duarte-Oden [7].

Let $Q^{N}$ denote an arbitrarily chosen set of $N$ points $x_{\alpha} \in \bar{\Omega}$ referred to as nodes:

$$
Q^{N}=\left\{x_{1}, x_{2}, \ldots, x_{N}\right\}, \quad x_{\alpha} \in \bar{\Omega} .
$$

Let $\mathcal{I}_{N}:=\left\{\omega_{\alpha}\right\}_{\alpha=1}^{N}$ denote a finite open covering of $\bar{\Omega}$ such that $x_{\alpha} \in \omega_{\alpha}, \alpha=1, \ldots, N$, and let $\mathcal{S}_{N}:=\left\{\mathcal{W}_{\alpha}\right\}_{\alpha=1}^{N}$ be a partition of unity subordinate to $\mathcal{I}_{N}$, that is, $\mathcal{S}_{N}$ is class of functions with the following properties:

$$
\begin{aligned}
& \mathcal{W}_{\alpha} \in C_{0}^{\infty}\left(\mathbb{R}^{n}\right), \\
& \operatorname{spt}\left(\mathcal{W}_{\alpha}\right)=\bar{\omega}_{\alpha}, \\
& \mathcal{W}_{\alpha}(x)>0, \quad x \in \omega_{\alpha}, \\
& \sum_{\alpha=1}^{N} \mathcal{W}_{\alpha}(x)=1, \quad \forall x \in \bar{\Omega} .
\end{aligned}
$$

In particular, for every $x \in \bar{\Omega}$, there is at least one $\mathcal{W}_{\beta}$ such that $\mathcal{W}_{\beta}(x)>0$. 
We remind that a basis of $\mathcal{F}^{0, k}$ is given by the functions

$$
\left\{\mathcal{W}_{\alpha} P_{\alpha, k}\right\}
$$

where $P_{\alpha, k} \in \mathcal{P}_{\alpha}^{k}$ and $\mathcal{P}_{\alpha}^{k}$ denotes the vector space of $k$-Taylor polynomials at $x_{\alpha}$

$$
\mathcal{P}_{\alpha}^{k}:=\left\{Q: Q(x)=\sum_{0 \leq|\nu| \leq k} a_{\nu}\left(x-x_{i}\right)^{\nu}\right\} .
$$

See [7] for details.

Let $\mathcal{F}_{\partial}$ be some finite dimensional vectorial space of functions over $\partial \Omega$. To simplify we assume that $\mathcal{F}_{\partial} \subset$ $C^{\infty}(\partial \Omega)$. The (Ritz-Galerkin) discretization of problem (4.6) is:

find $\left(u_{a}, \lambda\right) \in \mathcal{F}^{0, k} \times \mathcal{F}_{\partial}$, such that

$$
\widetilde{\mathcal{B}}\left(\left(u_{a}, \lambda\right),(v, \mu)\right)=\int_{\Omega} f v \mathrm{~d} x-\langle\langle\mu, g\rangle\rangle, \quad \forall(v, \mu) \in \mathcal{F}^{0, k} \times \mathcal{F}_{\partial} .
$$

Let $\left(\phi_{i}\right)_{i=1, \ldots, \operatorname{dim}\left(\mathcal{F}^{0, k}\right)}$ and $\left(\lambda_{q}\right)_{q=1, \ldots, \operatorname{dim}\left(\mathcal{F}_{\partial}\right)}$ basis of $\mathcal{F}^{0, k}$ and $\mathcal{F}_{\partial}$ respectively. The matrix associated to problem (5.1) is

$$
A=\left(\begin{array}{cc}
B & C^{T} \\
C & 0
\end{array}\right),
$$

where $B=\left(\mathcal{B}\left(\phi_{i}, \phi_{j}\right)\right)_{i=1, \ldots, \operatorname{dim}\left(\mathcal{F}^{0, k}\right)}$ and $C=\left(-\left\langle\left\langle\lambda_{q}, \psi\left(\phi_{j}\right)\right\rangle\right\rangle\right)_{q=1, \ldots, \operatorname{dim}\left(\mathcal{F}_{\partial}\right) ; j=1, \ldots, \operatorname{dim}\left(\mathcal{F}^{0, k}\right)}$. Then, although general, the method of Lagrange multipliers has the drawback of rendering a non-positive definite system of equations in addition to increasing the number of unknowns in $\operatorname{dim}\left(\mathcal{F}_{\partial}\right)$. However, this non-positive definite system of equations can be solved without major difficulties using, for example, a qmres iterative scheme provides that matrix $C$ behaves well. With this we mean that $C$ has a $\operatorname{dim}\left(\mathcal{F}_{\partial}\right) \times \operatorname{dim}\left(\mathcal{F}_{\partial}\right)$ positive definite submatrix with a good condition number. This problem is related to the choice of the space $\mathcal{F}_{\partial}$.

A thorough treatment of this question will be deferred to a forthcoming paper [15], although a brief description of the main ideas involved in our approach is as follows:

Let $x_{\beta}$ be a node in $\partial \Omega, \mathbf{n}_{\beta}$ the normal vector to $\partial \Omega$ at $x_{\beta}$. We choose a coordinate system $\left(y_{1}, \ldots, y_{n}\right)$ in a neighborhood of $x_{\beta}$ such that, in this coordinate system, $x_{\beta}=0$ and $\mathbf{n}_{\beta}=(1,0, \ldots, 0)$. We define the vectorial space $\mathcal{F}_{\beta, 0}^{0, k} \subset C^{\infty}(\partial \Omega)$ as that generated by the functions

$$
\left\{\mathcal{W}_{\beta} P_{\beta, k} \mid \partial \Omega\right\}
$$

where $P_{\beta, k} \in \mathcal{P}_{\beta}^{k}(2: n)$ and $\mathcal{P}_{\beta}^{k}(2: n)$ is the vector space of $k$-Taylor polynomials at 0 in the variables $y_{2}, \ldots, y_{n}$ and, for $i=1, \ldots, m-1, \mathcal{F}_{\beta, i}^{0, k} \subset C^{\infty}(\partial \Omega)$ is the vectorial space generated by the functions

$$
\left\{\xi y_{1}^{i}: \xi \in \mathcal{F}_{\beta, 0}^{0, k}\right\}
$$

For $i=0, \ldots, m-1$, we can now define

$$
\mathcal{F}_{\partial, i}:=\bigcup_{x_{\beta} \in \partial \Omega} \mathcal{F}_{\beta, i}^{0 . k}
$$

and

$$
\mathcal{F}_{\partial}:=\mathcal{F}_{\partial, 0} \cup \ldots \cup \mathcal{F}_{\partial, m-1} .
$$

Certainly, we could begin with smaller spaces than $\mathcal{F}_{\beta, 0}^{0, k}$ using, for example, only functions $\mathcal{W}_{\beta}$, in order not to increase the number of unknowns considerably, but the choice of the space $\mathcal{F}_{\partial}$ is strongly related to the quality of the approximations $\psi_{i} u_{a}$ to $g_{i}, i=0, \ldots, m-1$, in $\partial \Omega$. In a certain sense, Lagrange multipliers method is 
like a $L^{2}(\partial \Omega)$-projection method. If $m=1$ and the boundary condition is homogenous for example, $\psi_{0} u_{a}$ is orthogonal (in the $L^{2}(\partial \Omega)$ sense) to $\mathcal{F}_{\partial, 0}$. Then, more complete spaces $\mathcal{F}_{\partial, 0}$ produce better approximate results. In particular, in a Dirichlet problem $(m=1)$, even if the functions $\mathcal{W}_{\alpha}$ satisfy the Kronecker delta property, the Lagrange method takes more advantage of the spectral nature of the $h$ - $p$ clouds functions.

We have presented the major issues that appear in the numerical implementation of Lagrange multipliers. These kinds of problems will be further discussed as well as more extensive tests on the numerical properties of the proposed method will be presented in a forthcoming paper [15]. However, as a simple example, we will present a result related to the elasticity bilinear form (3.1) with $\nu=1 / 2$ over uniform grids of size $h$ in $\Omega=[0,1] \times[0,1]$. The boundary conditions are:

$$
\begin{aligned}
& \Psi_{0} u=u \mid \partial \Omega=0, \\
& \Psi_{1} u=\frac{\partial u}{\partial n}=0
\end{aligned}
$$

and $f$ is such that the exact solution of the problem is

$$
u(x, y)=\frac{\mathrm{e}^{200 x y(1-x)(1-y)}}{1-\mathrm{e}^{200 / 16}} .
$$

We have used a four point and a two point Gauss's formulas in the interior and in the boundary respectively. The Lagrange multipliers were built as described before.

Remark 5.1. Note that the families $\mathcal{F}^{0, k}$ reproduce polynomials of degree 1 and the bilinear form (3.1) is coercive only in subspaces $V$ which do not contain polynomials of degree 1 except, of course, $P=0$. However, there is no problem in the Lagrange multipliers formulation.

The different error measures evaluated are summarized below:

$$
\begin{gathered}
e r_{L^{2}, k}=\log \left(\frac{1}{\max _{\alpha}\left|u\left(x_{\alpha}\right)\right|} \sqrt{\frac{1}{N}\left(u\left(x_{\alpha}\right)-u_{h}\left(x_{\alpha}\right)\right)^{2}}\right), \\
e r_{L^{\infty}, k}=\max _{\alpha}\left|u\left(x_{\alpha}\right)-u_{h}\left(x_{\alpha}\right)\right| .
\end{gathered}
$$

The subscript $k$ means that the family $\mathcal{F}^{0, k}$ was used.

Convergence logarithm results are shown in the table below.

\begin{tabular}{|l|l|l|l|l|}
\hline$h$ & $e r_{L^{2}, 1}$ & $e r_{L^{\infty}, 1}$ & $e r_{L^{2}, 2}$ & $e r_{L^{\infty}, 2}$ \\
\hline \hline 0.125 & -4.7906 & 0.1715 & -8.0653 & 0.0096 \\
\hline 0.0625 & -7.3048 & 0.0272 & -9.2233 & 0.0030 \\
\hline 0.03125 & -8.8505 & 0.0138 & -11.757 & 0.0004 \\
\hline
\end{tabular}

The results show the good convergence properties of the method.

\section{REFERENCES}

[1] S. Agmon, Lectures on elliptic boundary value problems. D. Van Nostrand, Princeton, N. J. (1965).

[2] I. Babuška, The finite element method with lagrange multipliers. Numer. Math. 20 (1973) 179-192.

[3] I. Babuška and A.K. Aziz, Survey lectures on the mathematical foundations of the finite element method, The Mathematical Foundations of the Finite Element Method with Application to Partial Differential Equations. Academic Press, New York (1972) 5-359.

[4] T. Belytschko, Y. Krongauz. D. Organ, M. Fleming and P. Krysl, Meshless methods: an overview and recent development. Comput. Methods Appl. Mech. Engrg. 139 (1996a) 3-47. 
[5] J.M. Berezanskii, Expansions in Eigenfunctions of Self-Adjoint Operators, Translations of Mathematical Monographs 17, American Mathematical Society, Providence, R.I. (1968).

[6] S.C. Brener and L.R. Scott, The mathematical theory of finite elements methods. Springer-Verlag, New York (1994).

[7] C.A. Duarte and J.T. Oden, H-p clouds-an h-p meshless method. Num. Methods Partial Differential Equations. 1 (1996) $1-34$.

[8] S. Li and W.K. Liu, Meshfree and particle methods and their applications. Applied Mechanics Reviews (ASME) (2001).

[9] J.L. Lions and E. Magenes, Problèmes aux limites non homogènes et applications. Dunod, Paris (1968).

[10] G.R. Liu, Mesh Free Methods: Moving Beyond the Finite Element Method. CRC Press, Boca Raton, USA (2002).

[11] J. Nečas, Les méthodes directes en théorie des équations elliptiques. Masson, Paris (1967).

[12] J.T. Oden and J.N. Reddy, An introduction to the mathematical theory of finite elements. Wiley Interscience, New York (1976).

[13] K.T. Smith, Inequalities for formally positive integro-differential forms. Bull. Amer. Math. Soc. 67 (1961) 368-370.

[14] L.R. Volevič, Solvability of boundary value problems for general elliptic systems. Amer. Math. Soc. Transl. 67 (1968) $182-225$.

[15] C. Zuppa, G. Simonetti and A. Azzam, The h-p Clouds meshless method and lagrange multipliers for higher order elliptic operators. In preparation.

To access this journal online:

www.edpsciences.org 